\title{
GNSS RECEIVER ZERO BASELINE TEST USING GPS SIGNAL GENERATOR
}

\author{
Magdalena Dróżdż, Ryszard Szpunar \\ Department of Geodesy and Carography, Warsaw University of Technology \\ Pl. Politechniki 1, 00-661 Warsaw \\ m.drozdz@gik.pw.edu.pl,r.szpunar@gik.pw.edu.pl
}

\begin{abstract}
Validation of the performance of GPS receivers is crucial for many applications. This paper presents testing GPS receivers with the use of a GPS signal simulator. Unlike live testing, testing with simulators provides full control of simulated satellite signals and simulation environmental conditions. Moreover, generating repeatable signals makes possible to extend typical procedures. It allows to determine the absolute value of the position precision for each receiver without relation to any other one.
\end{abstract}

Keywords: baseline test, GPS signal generator, validation, GNSS receivers

\section{INTRODUCTION}

It is common known that satellite technologies dominate the geodesy market. Moreover, the GNSS technologies start to play a crucial role in many other application fields such as: navigation, aviation, logistics, localization, for both civilian and military purposes, Leick (1995). That creates the need for proper validation of GNSS receivers and systems. Testing of GNSS receivers in regard to a specific application should be an initial stage of each project. From economical prospective of companies it is more convenient to evaluate the receiver's performance under certain conditions before the complete utilization of the system. There is also the need to control the quality of the geodetic measurements. Accomplishment of that is impossible without proper validation and certification of receivers. Moreover, testing starts to become a crucial operation considering a growing supply of cheap GNSS receivers which are often provided without any detailed specification or form unreliable sources.

Unfortunately common tests using live signals can no longer be satisfactory. These tests can only provide limited information because the live signals are highly variable and nonrepeatable for a receiver. It is impossible to evaluate an absolute receiver's performance; the precision/accuracy of a particular instruments is always obtained with respect to the other receiver. Moreover, testing a receiver in different locations or under specific conditions is very unpractical and expensive. These limitations can solve a signal simulation. GPS signal simulator generates the same signals as those transmitted by GPS satellites, so the receiver processes them in exactly the same way. The advantage of this approach is that the specific set of signals can be presented to the receiver an unlimited number of times. Additionally, it is possible to control the signal processing environment and to determine the receiver's ability to work under various conditions, locations, times and movements. It makes possible to evaluate the receiver's performance in real conditions, its precision and accuracy, and also to determine its sensitivity to certain error sources and its software quality. The tests presented 
in this paper were carried out using live signals and those generated by the GSG-54 Pendulum GPS satellite simulator, and applied to different types of receivers.

\section{THE GPS SIGNAL SIMULATOR}

The GSG-54 Pendulum is an 8-chanell GPS constellation simulator (L1, C/A), so it provides an 8 satellites simulation. Moreover, it is possible to modify navigation data, antenna model, atmospheric model, multipath and more. The user can configure the pre-defined trajectories (static, circles, rectangular) by changing parameters such as user position and time, and upload own trajectory in the NMEA standard format. The simulator is also suited for accurate testing of timing GPS receivers, there is an input for external synchronization from e.g. a Cesium or Rubidium clock. It can also work as a pseudo-satellite.

\section{METHODOLOGY}

The field tests allow to examine the receiver's quality in particular span of time and under certain conditions. Unfortunately they do not provide information about the receiver's performance (software and hardware performance). An obtained error can be caused by software but also by influences of atmosphere, multipath or satellite constellation. The partial solution is the zero baseline test. In the zero baseline test, Szpunar et al. (2004), two or more receivers are connected to the same antenna/a signal simulator (Figure 1).

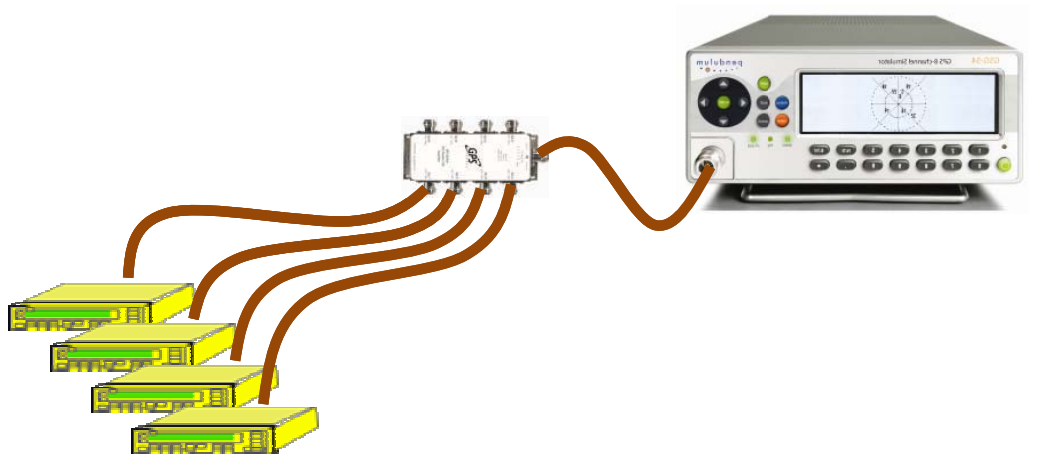

Fig.1. The configuration of receivers in the zero baseline test.

The test gives an impression of the observations, noise characteristics, since all common errors, like those due to multipath, atmosphere, satellite orbits and clocks are eliminated in the GPS baseline processing. But still the derived results can contain the impact of the geometry of the satellite constellation or the location of receiver, Parkinson et al. (1996). Moreover, the impression of the tested instrument's performance is always relative, in other words, with respect to the reference receiver. To overcome these disadvantages, laboratory tests should be conducted with help of the satellite signal simulator.

In proposed approach all receivers are connected to the same signal simulator (the zero baseline mode), so all of them process the same set of signals. The mentioned set of signals is presented to the receivers a few times (the measurement is repeated). In this way we obtain a 
few sessions of theoretically exactly the same observations (considering one receiver). Differencing observations between receivers but within the same session we can obtain the relative accuracy and precision. Analyzing observations of the one particular receiver but from all sessions allows to obtain the absolute accuracy and precision.

To track the receiver's performance with the time, the processing of observations is done in the kinematic mode. Due to the fact that the position is known in each epoch, the time series are created. The useful tool to generate the time series is the Trimble Total Control software (TTC). The kinematic processor in TTC performs the carrier phase reduction in two steps; the first step is called double difference float solution and derives baseline components and float phase ambiguities. The following step is ambiguity search and statistical tests like Fisher and Chi-square tests are used to verify the ambiguity resolution. In the GPS software Trimble Total Control is used the OTF mode in order to process the resolution, allowing the determination of ambiguities without any static initialization. The ambiguities are propagated throughout the whole tracking time of a satellite without loss of lock. In the last step the final carrier phase positions are computed, Trimble Total Control (2002).

\section{EXPERIMENTS}

Two Trimble 4700 receivers and two Leica GPS GRX1230GG receivers were used in experiments; for the sake of convenience, they are simply called: Trimble09, Trimble14, Leica06, Leica07. All measurements were performed in the zero-baseline scheme with the use of live and generated signals. All raw observations were processed with the help of the software Trimble Total Control in order to calculate coordinates of baselines in relation to a reference receiver. Obtained time series were filtered (the low pass filter - the moving average, the window 15). The receivers have been tested on two aspect - precision and accuracy.

\section{- Precision}

Firstly, the aim was to obtain results in terms of position repeatability, Szpunar et al. (2007). The measurements in a static scenario were carried out with the use of the GPS simulator. Five sessions were performed, all of them with the same ephemerides file on the $15^{\text {th }}$ January 2011 and with the same conditions applied to the simulator. In order to obtain an absolute position precision, for each receiver separately, four time series of zero-baselines were generated (sessions 2-5 were related to the first session). Figure 2 presents obtained results. To give the impression of satellite constellation's influence, PDOP coefficients versus time were also plotted there. 

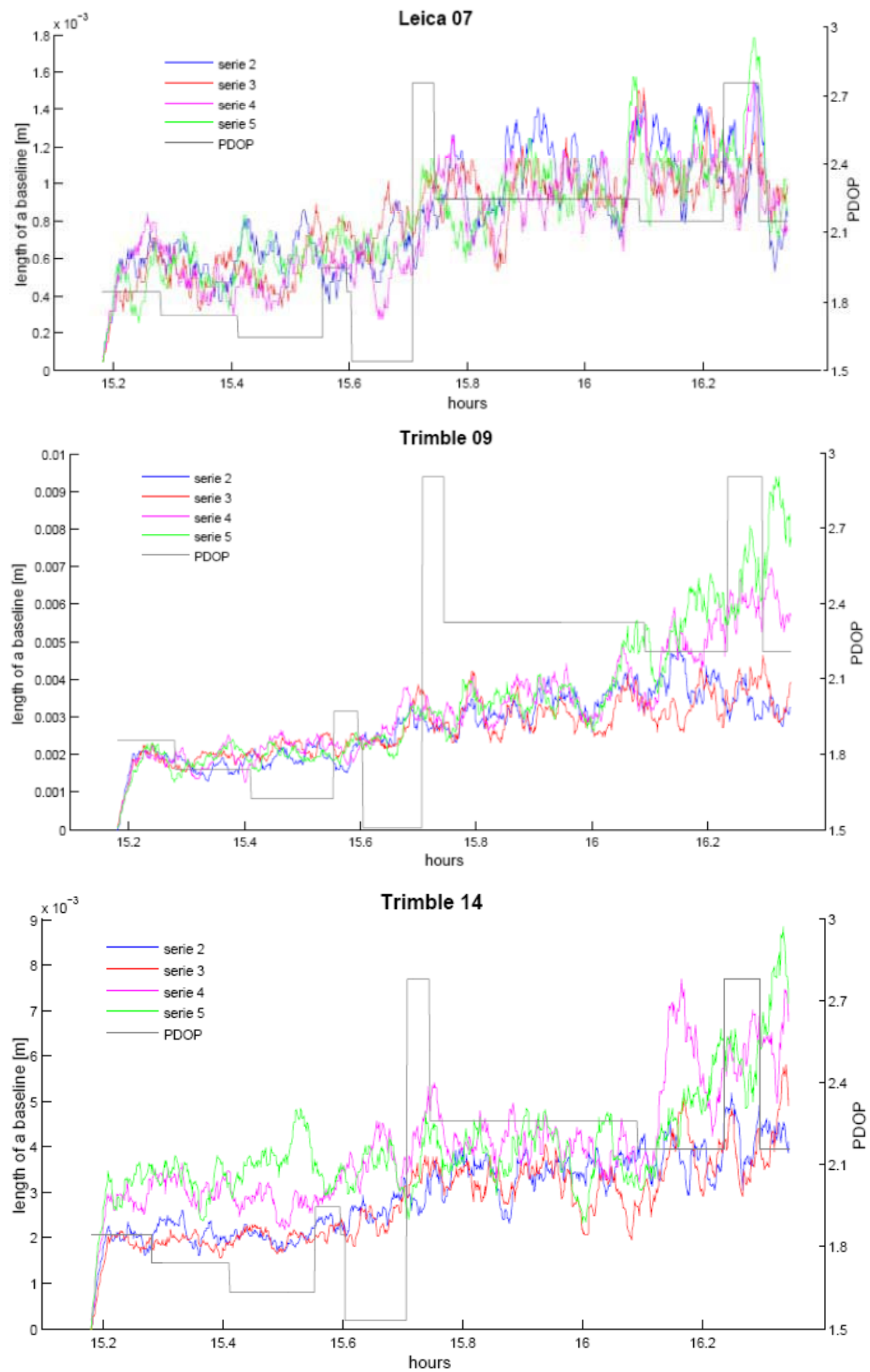

Fig. 2. Time series of zero baselines (color plots) and PDOP versus time; observations of each session related to observations of first session, separately for each receiver. 
To analyse the consilience between sessions and further to obtain conclusions about differences between receivers, correlation coefficients were calculated (Table 1). Firstly, correlation coefficients between all series (all possible 2-element permutations) and then an average coefficient for the pair of receivers were generated.

Table 1. Correlation coefficients for time series - precision.

\begin{tabular}{c|ccc} 
& L07 & T09 & T14 \\
\hline L07 & $\mathbf{0 , 8 5}$ & $\mathbf{0 , 7 2}$ & $\mathbf{0 , 6 3}$ \\
T09 & 0,72 & $\mathbf{0 , 8 6}$ & $\mathbf{0 , 7 5}$ \\
T14 & 0,63 & 0,75 & $\mathbf{0 , 8 3}$ \\
PDOP & $\mathbf{0 , 6 6}$ & $\mathbf{0 , 6 2}$ & $\mathbf{0 , 5 8}$
\end{tabular}

The auto-correlation coefficients of receivers are very similar. The lower consilience presents the Trimble14 receiver what is also seen in Figure 2. The highest cross-correlation coefficient have two Trimble receivers what is quite predictable. Values of cross-correlation coefficients between different receivers are very similar to cross-correlation coefficients between PDOP and receivers. This fact lets to conclude that the systematic part in coefficients is caused mainly by the geometry of satellite constellation. The another part is related to the repeatability of performance of each receiver.

Table 2. Statistical summary of results - precision.

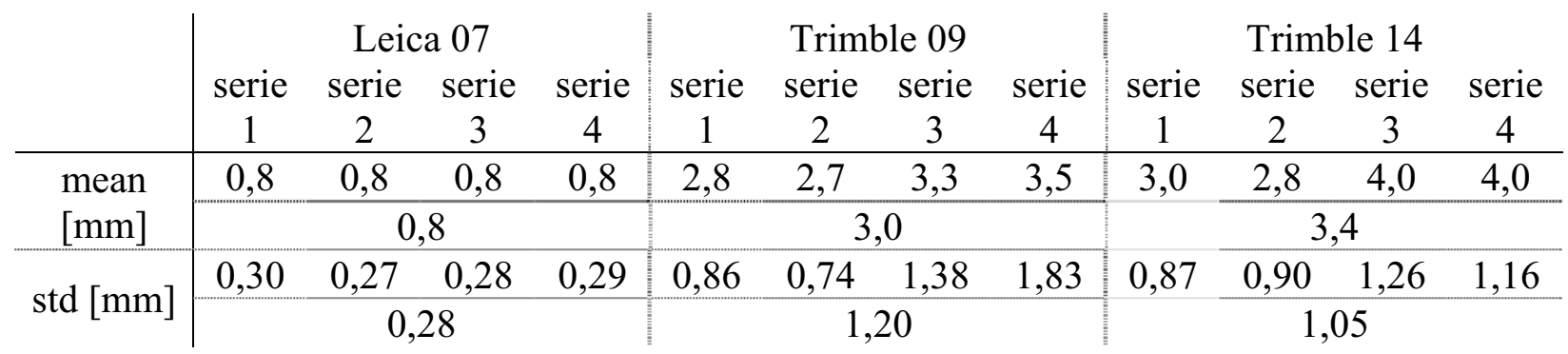

Table 2 contains the statistical summary of results. The mean values reflect the absolute position precision of each receiver. The newer Leica07 receiver presents significantly better quality than Trimble receivers.

- Accuracy

The aim of the second approach was to obtain some remarks about a relative accuracy of receivers. The quality of the Trimble 05 , the Trimble 14 and the Lecica06 receivers was assessed in the zero-baseline test in comparison with the Leica07 receiver. Time series of zero-baselines were generated separately for two sessions with the ephemerides file on the $15^{\text {th }}$ January 2011 (observations of the Leica07 receiver subtracted from observations of the other receivers, separately for each session). Results are shown in Fig. 3. Trimble receivers have pointed out more significant "sensitivity" to the bad geometry of the satellite constellation. 

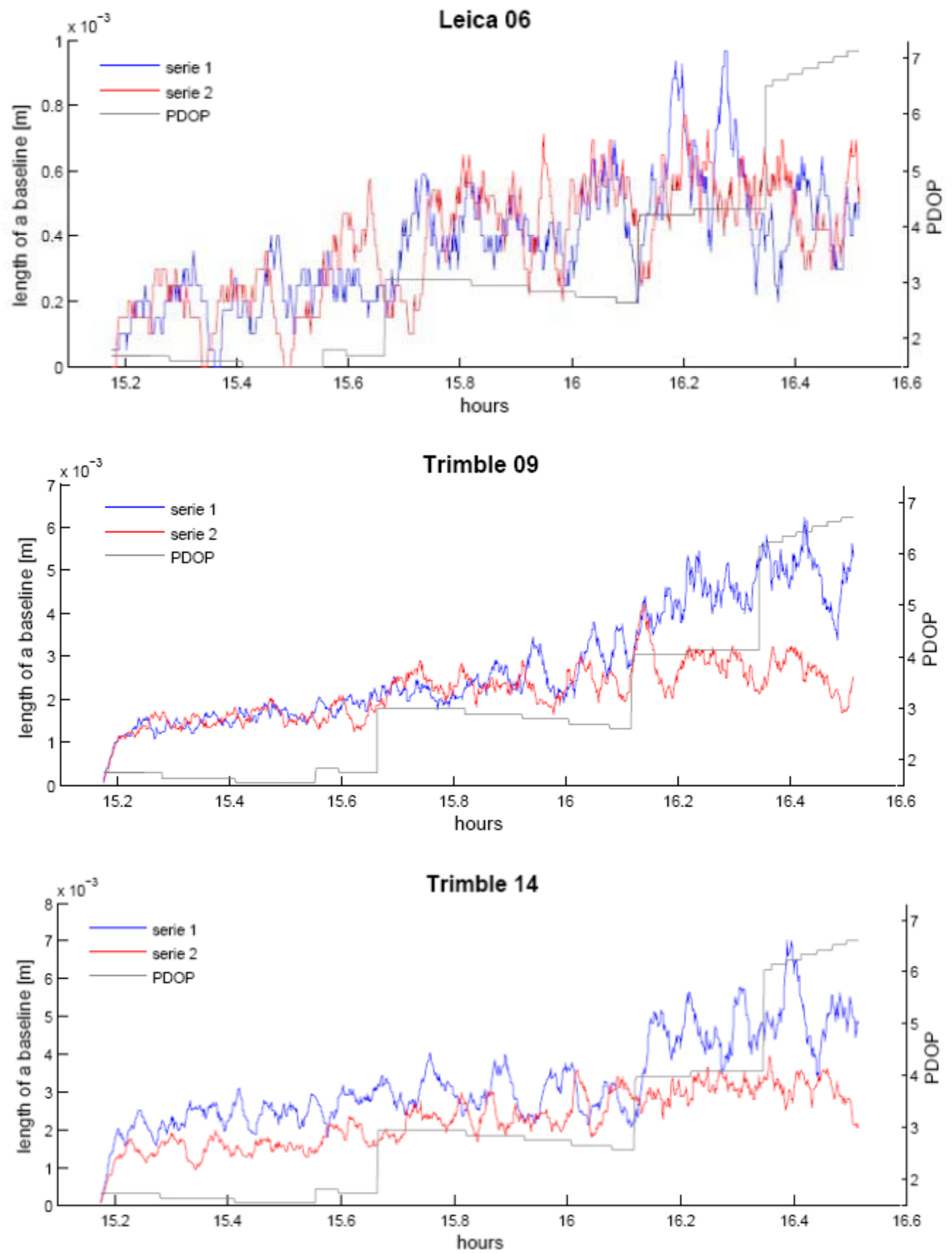

Fig. 3. Time series of zero baselines (color plots) and PDOP versus time, observations of each receiver related to the Leica07 receiver; separately for each session. 
Table 3. Statistical summary of results - accuracy.

\begin{tabular}{|c|c|c|c|c|c|c|}
\hline & \multicolumn{2}{|c|}{ Leica 06} & \multicolumn{2}{|c|}{ Trimble 09} & \multicolumn{2}{|c|}{ Trimble 14} \\
\hline & serie 1 & serie 2 & serie 1 & serie 2 & serie 1 & serie 2 \\
\hline \multirow{2}{*}{ mean $[\mathrm{mm}]$} & 0,4 & 0,4 & 2,8 & 2,1 & 3,3 & 2,3 \\
\hline & \multicolumn{2}{|c|}{0,4} & \multicolumn{2}{|c|}{2,5} & \multicolumn{2}{|c|}{2,8} \\
\hline \multirow{2}{*}{$\mathrm{std}[\mathrm{mm}]$} & 0,18 & 0,18 & 1,34 & 0,63 & 1,16 & 0,73 \\
\hline & \multicolumn{2}{|c|}{0,2} & \multicolumn{2}{|c|}{1,0} & \multicolumn{2}{|c|}{0,9} \\
\hline
\end{tabular}

It appears clearly (Table 3), that there is significant difference in the relative accuracy between Leica06 and two Trimble receivers. The evaluation was made under the assumption that the Leica07 receiver is the best quality. So it seems natural that the Leica06 presents better results with the relation to the Leica07. It can lead to an incorrect impression that any other reference receiver would give different results. But in fact, the obtained above crosscorrelation coefficients between different types of receivers are very similar. So there is no significant difference in the way of performance between receivers. The type of receiver should not have an influence on results. The choice of the reference receiver was based only on the quality of receiver meaning mainly random errors. Within the tests basing on position repeatability the Leica receivers have proved to maintain the highest precision comparing to the Trimble receivers. Due to that fact, one of the Leica receivers - the Leica07 receiver has been chosen as the reference receiver for analyzing the accuracy.

All experiments presented above were performed with the help of the signal simulator. To prove reliability of tests based only on simulated signals, the same calculations were executed but applying live and simulated signals. Within two sessions the live signal and the simulated signal were applied, both of them with exactly the same ephemeris file on the $8^{\text {th }}$ February 2011. Again, the quality of the Trimble05, the Trimble14 and the Lecica06 receivers was assessed in the zero-baseline test in comparison with the Leica07 receiver. Results are presented in Figure 4. 

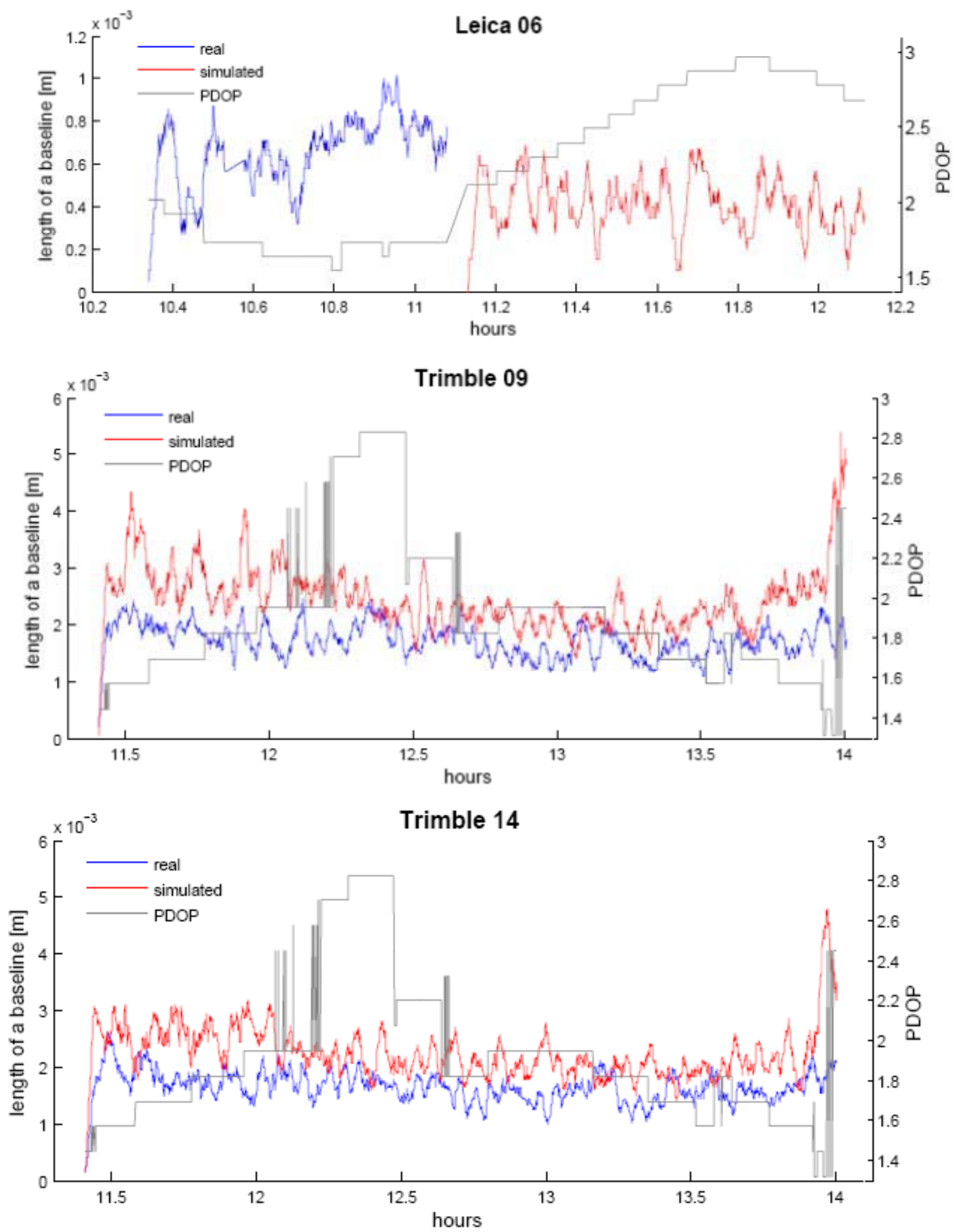

Fig. 4. Time series of zero baselines (color plots) and PDOP versus time, observations of all receivers related to the Leica07 receiver; separately for two series of live and simulated signal. 
Table 4. Statistical summary of results; live versus simulated signal.

\begin{tabular}{|c|c|c|c|c|c|c|}
\hline & \multicolumn{2}{|c|}{ Leica 06} & \multicolumn{2}{|c|}{ Trimble 09} & \multicolumn{2}{|c|}{ Trimble 14} \\
\hline & real & sim. & real & sim. & real & sim. \\
\hline \multirow{2}{*}{ mean $[\mathrm{mm}]$} & 0,7 & 0,4 & 1,7 & 2,5 & 1,7 & 2,3 \\
\hline & \multicolumn{2}{|c|}{0,5} & \multicolumn{2}{|c|}{2,1} & \multicolumn{2}{|c|}{2,0} \\
\hline \multirow{2}{*}{$\operatorname{std}[\mathrm{mm}]$} & 0,17 & 0,12 & 0,28 & 0,56 & 0,27 & 0,46 \\
\hline & \multicolumn{2}{|c|}{0,1} & \multicolumn{2}{|c|}{0,4} & \multicolumn{2}{|c|}{0,4} \\
\hline
\end{tabular}

The statistical summary presented in Table 4 shows that a way of signal generation does not have significant impact. Also in this approach, the Leica06 receiver has better relative accuracy. In case of the Trimble receivers, all values calculated with the help of the live signal are lower than the other based on the simulated signal. Probably it is caused by the bad signalto noise ratio (SNR) during the simulation. The variable which has to be implemented before simulation is the power level of the signal, the proper value of it plays a crucial role. During the experiment the choice of the power level was made basing on tests of a receiver's performance when applying different values of the power level. This is quite intuitive way, but the best possible solution at that moment, could lead to too noisy signal. Furthermore using splitter could have negative influence on survey by lowering the SNR ratio. But in case of the splitter used during these all experiments this impact is mitigated thanks to the construction of this splitter. The lowering the power level due to the splitting of signal is compensated by an amplifier.

In case of the Leica06 receiver, the results form simulation are better. The Leica06 is modern receiver, probably with better filters. But in fact, it is difficult to state if the receiver Leica06 was working properly. Obtained time series are very short (Figure 4); the time of experiment was more longer). So the results should not be trusted to make the final conclusions. More tests ought to be carried out.

To sum up, the Leica receivers have shown significantly better quality of performance than the Trimble receivers. Both analysis: precision and accuracy have proven the difference between these two types of receivers on level $2-3 \mathrm{~mm}$. That is probably mainly due to better filters and algorithms in case newer Leica receivers. This result seems reasonable in case of the kinematic solution wchich was applied within conducted experiments. But it should be pointed out that considering static solution a user should obtain values of accuracy in size of tenths of $\mathrm{mm}$.

\section{CONCLUSIONS}

The results derived from conducted experiments generate the new direction for the testing of receiver's performance. The results obtained when applying simulated signals are almost the same as these generated from live signals. So, the satellite signal simulator can be used for evaluation of the receiver's quality and performance. Moreover, applying the same signal a few times makes possible to extend typical procedures. It allows to determine the absolute 
value of position precision for each receiver without relation to any other one. This is not achievable when tracking only live signals.

The main advantage of the complementary approach (precision and accuracy) is that it makes possible to separate systematic and random errors. Comparing sessions of the same receiver gives the drawback about the random error and bugs in the receiver's software/hardware. Relating receivers within the one session allows to determine systematic errors in performance, but only under the assumption that the reference receiver works properly. Putting that all together provides full and more detailed information of the receiver's performance.

The disadvantage of the proposed methodology for observations processing is that the additional software (TTC) is used for calculating of the position in the kinematic mode. So, it must be taken into account that obtained results can be influenced by possible errors of this software. It suggests the need to process raw observations directly.

Our tests show that the modern Leica receivers present better quality than the Trimble receivers. They have better statistical summaries and also are less "sensitive" to the bad geometry of the satellite constellation and noisy signals.

\section{REFERENCES}

Leick A. (1995) GPS Satelite Surveying, Second Edition, A Wiley-Interscience Publication, New York.

Parkinson B.W., Spilker Jr. J.J. (1996) Global Positioning System: Theory and Applications, American Institute of Aeronautics and Astronautics, Washington.

Szpunar R., Walo J., Szelachowski W. (2004) Analysis of the permanent GPS observations applied for tracking the periodical changes of engineering objects, Reports on Geodesy, Vol. 69, No. 2, 329-334.

Szpunar R., Walo J. (2007) Displacement monitoring of engineering objects using GPS-RTK technique; EGU Congress "Geodetic and Geodynamic Programmes of the CEI" Vienna, Austria 15-20 April 2007, Reports on Geodesy, Vol. 83, No. 2, 83-87.

Trimble Total Control, User Guide, version 2.7,(2002).

Received: 2011-10-11,

Reviewed: 2012-02-09, by M. Figurski,

Accepted: 2012-02-16. 